Technical Report 1082

\title{
Night Vision Goggle Research and Training Issues for Ground Forces: A Literature Review
}

\author{
Jean L. Dyer \\ U.S. Army Research Institute \\ Keith M. Young \\ Aubum University \\ Consortium Research Fellows Program
}

\author{
Infantry Forces Research Unit \\ Scott E. Graham, Chief
}

\begin{abstract}
U.S. Army Research Institute for the Behavioral and Social Sclences
\end{abstract} 5001 Elsenhower Avenue, Alexandria, Virginia 22333-5600

\section{May 1998}

Approved for public release; distribution is unlimited. 